\title{
Lung scintigraphy in the diagnosis of pulmonary embolism: pathophysiological and practical evidence
}

\author{
Antonio Palla · Letizia Marconi · Francesca Bigazzi • \\ Massimo Pistolesi
}

Received: 18 July 2014/ Accepted: 13 September 2014/Published online: 27 September 2014

(C) Italian Association of Nuclear Medicine and Molecular Imaging 2014

\begin{abstract}
The protean clinical presentation of pulmonary embolism (PE) is the consequence of a complex series of haemodynamic and respiratory changes caused by the impact of thrombi within the pulmonary vasculature. Haemodynamic changes result from obstruction of the pulmonary arterial bed leading to increased pulmonary vascular resistances and they are manifested clinically as dyspnoea, engorgement of neck veins, and systemic arterial hypotension. Respiratory changes are characterised by lung volume reduction, hypocapnia and hypoxaemia. Reduced lung volume is mainly due to pulmonary consolidation, atelectasis and decreased distension of the thoracic cage due to chest pain. These pathophysiological changes affect both perfusion and ventilation and could explain the low sensitivity of the ventilation/perfusion (V/Q) mismatch scintigraphic approach in diagnosing PE. Acute PE causes redistribution of pulmonary blood flow from non-perfused areas and, to a lesser extent, of ventilation; this is a further factor arguing against the use of V/Q mismatch for the scintigraphic diagnosis of the disease. Hypoxaemia is primarily the consequence of V/Q abnormalities, namely the development of areas with a low V/Q ratio. On a Q scan this can easily be appreciated from hyperperfused lung
\end{abstract}

A. Palla $\cdot$ L. Marconi

CardioThoracic and Vascular Department, Section of Pulmonary

Diseases, University of Pisa, Pisa, Italy

A. Palla $(\square)$

Dipartimento Cardiotoraco Vascolare, Ospedale Cisanello,

Edificio 10, Via Paradisa 2, 56124 Pisa, Italy

e-mail: antonio.palla@med.unipi.it

F. Bigazzi - M. Pistolesi

Department of Experimental and Clinical Medicine,

Section of Respiratory Medicine, University of Florence,

Florence, Italy areas that, by themselves, are a marker for the scintigraphic diagnosis of PE. In conclusion, a Q scan without a V scan, when properly interpreted according to the prospective investigative study of acute pulmonary embolism diagnosis methodology (presence or absence of wedge-shaped perfusion defects) and combined with the formulation of a pretest clinical probability, can be used in most patients with clinical suspicion of PE, reducing costs and radiation load, increasing the practicality of the examination, and providing diagnostic accuracy comparable to that of CT angiography.

Keywords Pulmonary embolism - Clinical assessment . Instrumental diagnosis

\section{Introduction}

The diagnosis of pulmonary embolism (PE) based only upon clinical assessment is unreliable and thus impractical. However, clinical symptoms and signs are useful in formulating the pre-test clinical probability of $\mathrm{PE}$, thereby increasing the diagnostic accuracy of whatever imaging technique is used [1]. Therefore, the use of clinical assessment is recommended by international guidelines [14].

The instrumental diagnosis of PE has, for years, been made using ventilation/perfusion (V/Q) scintigraphy. This diagnostic approach was developed on the basis of the theoretical expectation that regions of the lung excluded from perfusion by emboli maintained normal ventilation, thus giving rise to mismatched patterns of perfusion and ventilation [5]. Nowadays, this nuclear medicine methodology is still employed, although less so than in the recent past. Unfortunately, the assumption of mismatched 
perfusion and ventilation conflicts with the pathophysiological notion of ventilation redistribution away from lung regions in which pulmonary artery circulation has been acutely obstructed [6]. Accordingly, a high frequency of non-diagnostic or false-negative readings of V/Q examinations was demonstrated in the first prospective investigation on pulmonary embolism diagnosis (PIOPED I) study [7]. As a consequence, the use of the V/Q scan has declined in favour of the more accurate and more readily available pulmonary CT angiography (CTA) examination. However, CTA also suffers limitations mostly due to its considerable radiation burden, high costs, and contraindications in a considerable portion of patients. Therefore, a reappraisal of lung scintigraphy in the diagnosis of PE is warranted.

It has recently been demonstrated that a perfusion (Q) scan without ventilation (V) has a diagnostic accuracy in PE that is comparable to that of CTA [8]. The Q scan could thus be considered as an alternative approach to CTA for diagnosing acute PE, especially in young women or in patients with contraindications to contrast media. Accordingly, the most recent Society of Nuclear Medicine guidelines includes the $\mathrm{Q}$ scan without the $\mathrm{V}$ scan among the recommended imaging methodologies for diagnosing acute PE [9].

The aim of this paper is to review the most relevant pathophysiological notions underlying the clinical presentation of PE and to describe their significance in relation to different diagnostic approaches, specifically the V/Q scan and the Q scan.

\section{Pathophysiological mechanisms underlying the clinical presentation of $P E$ and practical implications for the V/Q scan diagnostic approach}

Depending upon the extent of pulmonary vascular bed occlusion, the cardiopulmonary status before PE, and the time elapsed between embolisation and diagnosis, patients with acute PE may have a wide spectrum of clinical presentations, ranging from mild non-specific symptoms to acute cor pulmonale and sudden death. The clinical manifestations of acute PE are therefore variable although it is usually accepted that the great majority of patients complain of sudden-onset dyspnoea, chest pain and fainting $[10,11]$.

Hypoxaemia resulting from alterations in the V/Q relationship is the main factor responsible for acute dyspnoea in PE.

The multiple inert gas elimination technique (MIGET), based on analysis of the concentrations of six inert gases in venous and arterial blood and in expired air, has been applied to determine the origin of hypoxaemia in $\mathrm{PE}$ [12]. In a normal subject, V/Q distribution displays two curves, the peaks of which are superimposed. Patients with acute PE present a broad spectrum of V/Q inequalities. The most frequent pattern is the development of regions of high V/Q ratio, where perfusion is markedly reduced while ventilation is still present, although reduced. Some regions have an abnormally low V/Q ratio where perfusion is increased with respect to normal (areas free from emboli where perfusion is diverted forcedly). Such regions with abnormally low V/Q ratio receive as much as $20 \%$ of cardiac output [13]. The pattern of $\mathrm{V} / \mathrm{Q}$ inequalities in patients with acute PE may also be evaluated by studying, using nuclear medicine techniques, the regional distribution of ventilation and perfusion. Indeed, the $\mathrm{Q}$ scan demonstrates extensive perfusion defects, but also areas with forced perfusion where ventilation is also diverted but to a smaller extent (low V/Q ratio) [13]. It is the areas with low V/Q ratio (and not the embolised regions, as is widely held) that are mostly responsible for the development of hypoxaemia [13]. Furthermore, the above observations imply a redistribution of ventilation away from the embolised lung regions and this clearly conflicts with the assumption underlying the use of the V/Q strategy. Indeed, ventilation and perfusion are redistributed to about the same extent in most cases of acute PE. This is perhaps the main reason why the PIOPED I study authors reported an unacceptably low level of sensitivity for diagnosis of $\mathrm{PE}$ with the V/Q scan [7, 14].

Redistribution of ventilation may occur by different mechanisms in the various stages of PE. This was clearly demonstrated in man in the very acute stage of PE by Santolicandro et al. [13] using the MIGET technique, but it was known even before MIGET became available. Levy and Simmons [15] suggested that a ventilation shift occurred following thromboembolisation. This early shift of ventilation away from the embolised lung regions may be mediated by local hypocapnia, which occurs immediately after pulmonary arterial occlusion [16]. It has been shown in healthy humans that hypocapnia can induce marked bronchoconstriction and possibly also an increase in lung tissue resistance [17]. These effects are ascribed to the direct action of carbon dioxide because they can be also observed in isolated preparations of airway smooth muscle tissue [18]. All the above observations may also explain why hypocapnia might be a transient instrumental finding characterising the first days after embolisation, and tending to return toward normal values in the following days [19].

At a later stage, over a time span ranging from several hours to a few days, the ventilation shift away from embolised lung regions may occur by different mechanisms, such as alterations of surfactant production and small airway obstruction. A decrease in surface-active material may occur distally to the occluded vessels after a relatively long interval (18-24 h) following experimental embolisation, 
and it is considered to be the cause of the haemorrhagic atelectasis often seen after acute pulmonary thromboembolism [20]. These experimental observations may also explain the commonly observed radiographic finding of lung volume reduction in subjects with PE. It is commonly known that several radiographic findings such as consolidation (with or without true infarction), plate-like atelectasis, elevated diaphragm, and pleural effusion are frequently observed in acute PE. Their occurrence is another major cause of the low sensitivity of the V/Q scan. In fact, they were observed in $88 \%$ of patients in the PIOPED I study [14] and in $86 \%$ of the patients included in the prospective investigative study of acute pulmonary embolism diagnosis (PISA-PED) [21]. Furthermore, retrospective examination of the conventional pulmonary angiograms obtained in the PIOPED I study demonstrated the presence of consolidation or atelectasis in about $50 \%$ of the lung regions with angiographic filling defects [7]. On the whole, such radiographic abnormalities further explain the inconsistency of the diagnostic strategy based on V/Q mismatch. Indeed, the above radiographic changes imply a ventilation shift away from the embolised lung regions; a V scan cannot be normal in embolised areas where consolidation or atelectasis coexists. When such coexistence occurs, a V/Q mismatch is no longer easily detected and, consequently, the level of sensitivity of the diagnostic strategy is low. Consequently, it is not surprising that, adopting the criterion of V/Q mismatch for the scintigraphic diagnosis of $\mathrm{PE}$, the sensitivity of the procedure (high-probability lung scan) was only $41 \%$ in the original PIOPED I study [7]. This unacceptably low level of sensitivity for this diagnostic strategy is exactly as expected considering the frequency of the mentioned chest radiographic findings and it is an important factor arguing against the use of the V scan in addition to the Q scan, which is what was originally suggested [5].

All the above considerations had been clearly foreseen by the pulmonary physiologist, Comroe [6], who in 1966, stated: "to the surprise of no one, occlusion of one pulmonary artery for 24,48 , or $72 \mathrm{~h}$ led to a decrease in ventilation to the lung, but to the surprise of everyone, this lung was atelectatic and markedly congested" and concluded that "the decrease in wasted ventilation (ventilation to non-perfused and poorly perfused lung) helps the patients but hinders the physician in diagnosis". Unfortunately, these insightful conclusions were ignored for years by both radiologists and nuclear medicine physicians.

Hypocapnia is also a potential cause of dyspnoea. The pathogenesis of hypocapnia is due to the increment of total and alveolar ventilation, caused by the onset of hypoxaemia on the one hand, and stimulation of lung mechanical receptors on the other [18, 22]. This increment occurs in spite of the increase in physiological dead space caused by ventilation of non-perfused pulmonary units. Therefore, whatever measurement technique is employed, the increase in dead space is small due to the redistribution of ventilation towards perfused areas, which reduces the amount of wasted ventilation. The small increase in dead space also suggests that, in most patients with acute PE, vascular obstruction is incomplete; this is confirmed by perfusion lung scintigraphy that usually shows zones of residual blood flow in embolised areas [23]. These observations may thus provide an explanation for the inappropriateness of dead space measurement in the diagnosis of PE, since the increase in dead space, although present, greatly underestimates the percentage of unperfused alveoli [24].

In conclusion, all the above pathophysiological considerations demonstrate that even though the V/Q scan is still the nuclear medicine technique most widely employed to diagnose PE, the contemporaneous use of both $\mathrm{V}$ and $\mathrm{Q}$ scintigraphy has theoretical drawbacks.

\section{Pathophysiological mechanisms underlying the clinical presentation of $P E$ and practical implications for the $\mathbf{Q}$ scan diagnostic approach}

For years, perfusion scintigraphy has been considered highly sensitive (about $100 \%$ ) but poorly specific in the diagnosis of $\mathrm{PE}$; this is because many lung pathologies may cause perfusion defects mimicking the presence of emboli. As shown in the above section, the addition of a $\mathrm{V}$ scan to improve $Q$ scan specificity has significant drawbacks. An alternative could be to use different methodologies to perform the Q scan, such as the SPECT technique, which is still under investigation (see the related paper in this issue of Clinical and Translational Imaging).

Substantial progress towards increasing the specificity of perfusion scintigraphy was made with the introduction, about two decades ago, of a new simplified method of evaluating the presence of pulmonary perfusion defects, which made it easier to attribute the defect to PE instead of other lung diseases [21]. In more detail, pulmonary perfusion was interpreted for the first time contemporaneously with chest X-ray and the perfusion defects were classified on the basis of their shape (wedge-shaped or not), independently of their number. Accordingly, pulmonary perfusion was classified as normal (absence of defects), almost normal (defects due to heart or hilar vessels, elevated diaphragm, as judged on the basis of X-ray), abnormal and compatible with PE (single or multiple wedge-shaped defects), abnormal and not compatible with PE (non wedge-shaped defects or defects clearly explained by radiographic abnormalities).

Moreover, in the same years, the pre-test clinical probability of PE was introduced into clinical practice as an 
essential method for increasing the diagnostic accuracy of whatever chest imaging technique is used to diagnose PE [1]. In this regard, the PIOPED II study demonstrated that even CTA, usually considered the new gold standard [25], shows low accuracy in the presence of results conflicting with the pre-test clinical probability [26]. In fact, in patients with a positive CTA and a low clinical probability and in those with a negative CTA and high clinical probability, the CTA diagnosis does not have sufficient positive or negative diagnostic predictive value to allow a correct clinical decision on whether to start or withhold anticoagulant treatment.

Therefore, the pre-test clinical probability assessment was recommended by the most important scientific societies as a prerequisite for diagnosis.

Three original methods have been proposed and tested in large-scale clinical trials [27-29]. All methods work efficiently in excluding $\mathrm{PE}$ in patients in the emergency department, while the method developed by the PISA-PED group [27] appears to be the most efficient in correctly establishing a high or low clinical probability of PE in both in- and outpatients [30]. Once again, knowledge of the pathophysiology underlying the clinical manifestation of PE is essential to judge the efficiency of the methods used to establish PE clinical probability. The PISA-PED methodology, at variance with the other two methods [28, 29], included among the criteria for establishing probability not only the most relevant symptoms (dyspnoea, chest pain, syncope) but also the findings of simple, non-invasive techniques (ECG, chest X-ray). Although the methods developed by Swiss and Canadian authors [28, 29] are the most widely used worldwide due to their easier applicability, they do not take into consideration the above symptoms and signs and are less accurate in the formulation of pre-test clinical probability [30].

By establishing pre-test probability assessed according to the PISA-PED model, which considers the most important symptoms and instrumental findings of PE, and combining this with $\mathrm{Q}$ scanning, it has been possible to develop a diagnostic strategy for PE that yields results comparable to those of CTA [27]. These findings were recently confirmed in a study in which the V/Q scans acquired in the PIOPED II study were read (without the V scan) according to the PISAPED methodology by two independent experts unaware of the final diagnosis. The $\mathrm{Q}$ scan positive and negative predictive values were found to be comparable to those of CTA with a lower number of non-diagnostic results [8].

\section{Conclusions}

In summary, a $\mathrm{Q}$ scan without a $\mathrm{V}$ scan, when properly interpreted and combined with the formulation of pre-test clinical probability, can be used in most patients with clinical suspicion of PE and could be the test of choice in young women and in patients with contraindications to CTA such as renal insufficiency and allergy to contrast media.

Conflict of interest Antonio Palla, Letizia Marconi, Francesca Bigazzi and Massimo Pistolesi declare that they have no conflict of interest.

Human and animal studies This article does not contain any studies with human or animal subjects performed by any of the authors.

\section{References}

1. British Thoracic Society Standards of Care Committee Pulmonary Embolism Guideline Development Group (2003) BTS guidelines for the management of suspected acute pulmonary embolism. Thorax 58:470-484

2. Stein PD, Sostman HD, Bounameaux H, Buller HR, Chenevert TL, Dalen JE, Goodman LR, Gottschalk A, Hull RD, Leeper KV Jr, Pistolesi M, Raskob GE, Wells PS, Woodard PK (2008) Challenges in the diagnosis of acute pulmonary embolism. Am J Med 121:565-571

3. Bajc M, Neilly JB, Miniati M, Schuemichen C, Meignan M, Jonson B, EANM Committee (2009) EANM guidelines for ventilation/perfusion scintigraphy: Part 1 . Pulmonary imaging with ventilation/perfusion single photon emission tomography. Eur J Nucl Med Mol Imaging 36:1356-1370

4. Torbicki A, Perrier A, Konstantinides S, Agnelli G, Galiè N, Pruszczyk P, Bengel F, Brady AJ, Ferreira D, Janssens U, Klepetko W, Mayer E, Remy-Jardin M, Bassand JP, ESC Committee for Practice Guidelines (CPG) (2008) Guidelines on the diagnosis and management of acute pulmonary embolism: the task force for the diagnosis and management of acute pulmonary embolism of the European society of cardiology (ESC). Eur Heart J 29:2276-2315

5. McNeil BJ, Holman BL, Adelstein SJ (1974) The scintigraphic definition of pulmonary embolism. JAMA 227:753-756

6. Comroe JH Jr (1966) The main functions of the pulmonary circulation. Circulation 33:146-158

7. Investigators PIOPED (1990) Value of the ventilation/perfusion scan in acute pulmonary embolism: results of the prospective investigation on pulmonary embolism diagnosis (PIOPED). JAMA 263:2753-2759

8. Sostman HD, Miniati M, Gottschalk A, Matta F, Stein PD, Pistolesi M (2008) Sensitivity and specificity of perfusion scintigraphy combined with chest radiography for acute pulmonary embolism in PIOPED II. J Nucl Med 49:1741-1748

9. Parker JA, Coleman RE, Grady E, Royal HD, Siegel BA, Stabin MG, Sostman HD, Hilson AJ; Society of Nuclear Medicine (2012) SMN practice guideline for lung scintigraphy 4.0. J Nucl Med Technol 40:57-65

10. Palla A, Petruzzelli S, Donnamaria V, Giuntini C (1995) The role of suspicion in the diagnosis of pulmonary embolism. Chest 107(1 Suppl):21S-24S

11. Miniati M, Cenci C, Monti S, Poli D (2012) Clinical presentation of acute pulmonary embolism: survey of 800 cases. PLoS ONE 7:e30891

12. Wagner PD, Lavaruso RB, Uhl RR, West JB (1974) Continuous distributions of ventilation-perfusion ratios in normal subjects breathing air and 100 per cent $\mathrm{O}_{2}$. J Clin Invest 54:54-68 
13. Santolicandro A, Prediletto R, Fornai E, Begliomini E, GiannellaNeto A, Giuntini C (1995) Mechanisms of hypoxemia and hypocapnia in pulmonary embolism. Am J Respir Crit Care Med 152:336-347

14. Worsley DF, Alavi A, Aronchick JM, Chen JT, Greenspan RH, Ravin CE (1993) Chest radiographic findings in patients with acute pulmonary embolism: observations from the PIOPED study. Radiology 189:133-136

15. Levy SE, Simmons DH (1974) Redistribution of alveolar ventilation following pulmonary thromboembolism in the dog. J Appl Physiol 36:60-68

16. Allgood RJ, Wolfe WG, Ebert PA, Sabiston DC Jr (1968) Effects of carbon dioxide on bronchoconstriction after pulmonary artery occlusion. Am J Physiol 214:772-775

17. D'Angelo E, Calderini IS, Tavola M (2001) The effects of $\mathrm{CO}_{2}$ on respiratory mechanics in anesthetized paralyzed humans. Anesthesiology 94:604-610

18. Twort $\mathrm{CH}$, Cameron IR (1986) Effects of $\mathrm{PCO}_{2}, \mathrm{pH}$, and extracellular calcium on contraction of airway smooth muscle from rats. Respir Physiol 66:259-267

19. Prediletto R, Paoletti P, Fornai E, Perissinotto A, Petruzzelli S, Formichi B, Ruschi S, Palla A, Giannella-Neto A, Giuntini C (1990) Natural course of treated pulmonary embolism. Evaluation by perfusion lung scintigraphy, gas exchange, and chest roentgenogram. Chest 97:554-561

20. Chernick V, Hodson WA, Greenfield LJ (1966) Effect of chronic pulmonary artery ligation on pulmonary mechanisms and surfactant. J Appl Physiol 21:1315-1320

21. Miniati M, Pistolesi M, Marini C, Di Ricco G, Formichi B, Prediletto R, Allescia G, Tonelli L, Sostman HD, Giuntini C (1996) Value of perfusion lung scan in the diagnosis of pulmonary embolism: results of the prospective investigative study of acute pulmonary embolism diagnosis (PISA-PED). Am J Respir Crit Care Med 154:1387-1393
22. Domino KB, Emery MJ, Swenson ER, Hlastala MP (1988) Ventilation heterogeneity is increased in hypocapnic dogs but not in pigs. Respir Physiol 111:89-100

23. Giuntini C (2001) Ventilation/perfusion scan and dead space in pulmonary embolism: are they useful for the diagnosis? Q J Nucl Med 45:281-286

24. Giuntini C, Santolicandro A, Prediletto R, Paoletti P, Formichi B, Formichi B, Fornai E, Begliomini E, Puntoni R, Perissinotto A, Giannella Neto A (1997) Acute respiratory failure: pulmonary thromboembolism. Monaldi Arch Chest Dis 52:566-569

25. Remy-Jardin M, Pistolesi M, Goodman LR, Gefter WB, Gottschalk A, Mayo JR, Sostman HD (2007) Management of suspected acute pulmonary embolism in the era of CT angiography: a statement from the Fleischner Society. Radiology 245:315-329

26. Stein PD, Fowler SE, Goodman LR, Gottschalk A, Hales CA, Hull RD, Leeper KV Jr, Popovich J Jr, Quinn DA, Sos TA, Sostman HD, Tapson VF, Wakefield TW, Weg JG, Woodard PK, PIOPEDII Investigators (2006) Multidetector computed tomography for acute pulmonary embolism. N Engl J Med 354:2317-2327

27. Miniati M, Prediletto R, Formichi B, Marini C, Di Ricco G, Tonelli L, Allescia G, Pistolesi M (1999) Accuracy of clinical assessment in the diagnosis of pulmonary embolism. Am J Respir Crit Care Med 159:864-871

28. Wicki J, Perneger TV, Junod AF, Bounameaux H, Perrier A (2001) Assessing clinical probability of pulmonary embolism in the emergency ward: a simple score. Arch Intern Med 161:92-97

29. Wells PS, Ginsberg JS, Anderson DR, Kearon C, Gent M, Turpie AG, Bormanis J, Weitz J, Chamberlain M, Bowie D, Barnes D, Hirsh J (1998) Use of a clinical model for safe management of patients with suspected pulmonary embolism. Ann Intern Med 129:995-1005

30. Eisner MD (2003) Before diagnostic testing for pulmonary embolism: estimating the prior probability of disease. Am J Med 114:232-234 